\title{
Classifications In Brief: Salter-Harris Classification of Pediatric Physeal Fractures
}

\author{
Daniel J. Cepela MD, Jason P. Tartaglione MD, Timothy P. Dooley MD, \\ Prerana N. Patel MD
}

Received: 9 November 2015/Accepted: 5 May 2016/Published online: 20 May 2016

(C) The Association of Bone and Joint Surgeons ( 2016

\section{History}

Fractures involving the epiphyseal plate, or physis, are common musculoskeletal injuries occurring in children with open growth plates. These fractures represent between $15 \%$ and $18 \%$ of all pediatric fractures [13, 24, 26] and present diagnostic and treatment challenges for orthopaedic surgeons. The first detailed description of injuries involving the epiphyseal plate was in 1863 by Foucher [8]. In 1898, Poland classified these fractures into four types [20]. Aitken further defined the characteristics of different types of physes with respect to structure, location, weightbearing status, and susceptibility to injury, suggesting that prognosis be considered on an individual basis [1]

In 1963, two Canadian orthopaedic surgeons, Robert B. Salter (1924-2010) and W. Robert Harris (1922-2005), created a physeal fracture classification system based on

Each author certifies that he or she, or a member of his or her immediate family, has no funding or commercial associations (eg, consultancies, stock ownership, equity interest, patent/licensing arrangements, etc) that might pose a conflict of interest in connection with the submitted article

All ICMJE Conflict of Interest Forms for authors and Clinical Orthopaedics and Related Research ${ }^{\circledR}$ editors and board members are on file with the publication and can be viewed on request.

This work was performed at Albany Medical Center, Albany, NY, USA

D. J. Cepela ( $\square)$, J. P. Tartaglione, T. P. Dooley

Department of Surgery, Albany Medical Center, 43 New

Scotland Avenue, Albany, NY 12208, USA

e-mail: dcepela@gmail.com

P. N. Patel

Shriners Hospital for Children, Springfield, MA, USA anatomy, fracture pattern, and prognosis [26]. Salter and Harris described two major types of epiphyseal plates: the pressure epiphysis and the traction epiphysis. Pressure epiphyses provide longitudinal growth, occur at the end of long bones, are intraarticular, and bear weight. In contrast, traction epiphyses provide appositional growth, occur at the origin or insertion of muscles, are extraarticular, and do not bear weight. Their classification focuses on injuries at pressure epiphyses. They recognized that physeal fractures consistently occur through the same histologic plane called the zone of provisional calcification (Fig. 1). This zone, located in the zone of hypertrophy, represents a transitional point between calcified and noncalcified extracellular matrix proteins, making it weaker than its surrounding osseous-ligamentous structures, and therefore more susceptible to injury.

Since the landmark paper by Salter and Harris [26], which characterized five types of physeal fractures (Fig. 2), various authors have expanded on the original work of Salter and Harris in attempts to be more comprehensive. Rang [21] added a sixth type of physeal injury which described damage to the perichondral ring that resulted from direct open injuries. Ogden [15] described nine types of injuries. This revised classification system included subclassifications of the original five Salter-Harris patterns meant to stratify the risk of growth arrest based on injury patterns. In addition, he described four new types that included injury to developing bone's other growth mechanisms (metaphyseal, diaphyseal, periosteal, zone of Ranvier, and epiphyseal perichondrium) not included in the Salter-Harris classification system. However despite multiple attempts to revise and expand on the classification of Salter and Harris, their system continues to be the mostwidely used classification system of physeal injuries. 


\section{Purpose}

An ideal fracture classification system should be reproducible, possess high inter- and intraobserver reliability, anticipate prognosis indicator, and guide clinical decisionmaking.

Physeal injuries have the potential for growth arrest and resulting deformity; however, not all injuries to the physis pose the same risk. Therefore, a classification system that is able to identify injury patterns that carry a high risk of physeal arrest and deformity would be desirable. Salter and

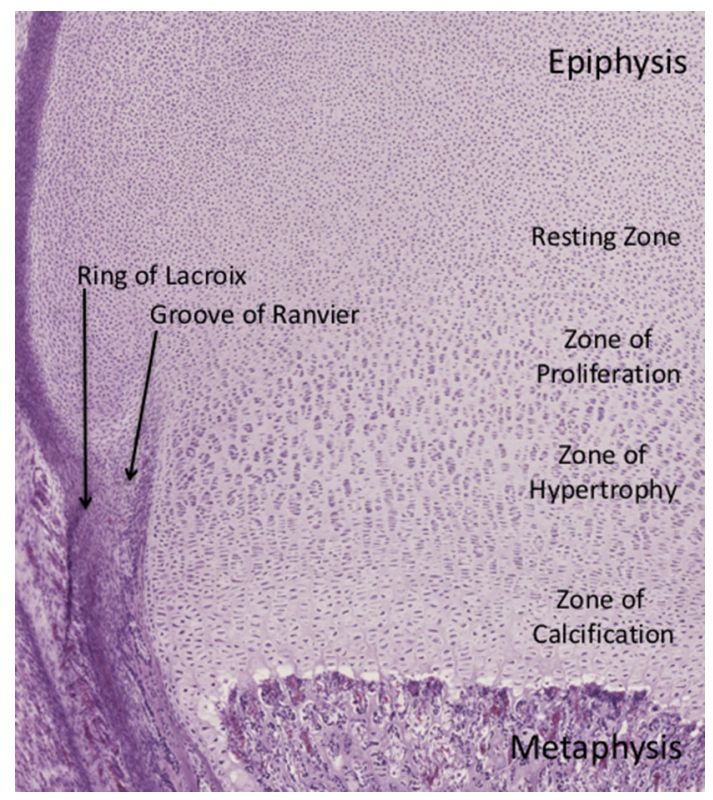

Fig. 1 The histology of the physis with standard hematoxylin and eosin staining is shown. The top of the slide is oriented toward the epiphysis while the bottom is oriented toward the metaphysis. The four zones of the physis and the surrounding fibrocartilaginous structures are shown. The most common region for physeal fractures to occur is through the zone of provisional calcification, which is a subzone of the zone of hypertrophy.
Harris' [26] originally stated purpose was the accurate description of physeal injuries and prognosis relating to premature physeal closure.

\section{Description}

The Type I fracture (Figs. 2, 3), as originally described by Salter and Harris [26], extends directly through the growth plate, resulting in separation of the epiphysis from the metaphysis. According to Salter and Harris, the Type I

\section{Salter-Harris Classification}

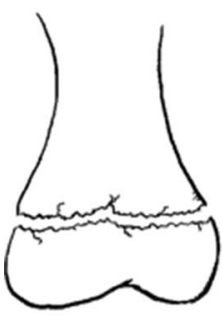

I

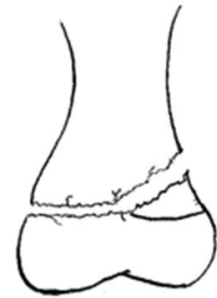

II

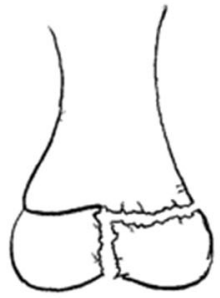

III

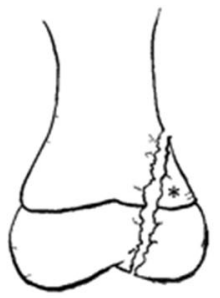

IV

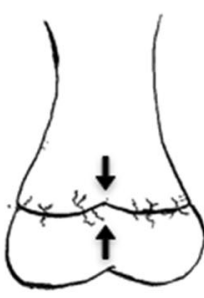

V
Fig. 2 The five basic fracture types of the Salter-Harris classification are shown. A Type I fracture is a separation through the physis. A Type II fracture enters in the plane of the physis and exits through the metaphysis. The resulting metaphyseal fragment is called the Thurston-
Holland fragment (*). A Type III fracture enters in the plane of the physis and exits through the epiphysis. A Type IV fracture crosses the physis, extending from the metaphysis to the epiphysis. A Type V fracture is a crush injury resulting in injury to the physis. 


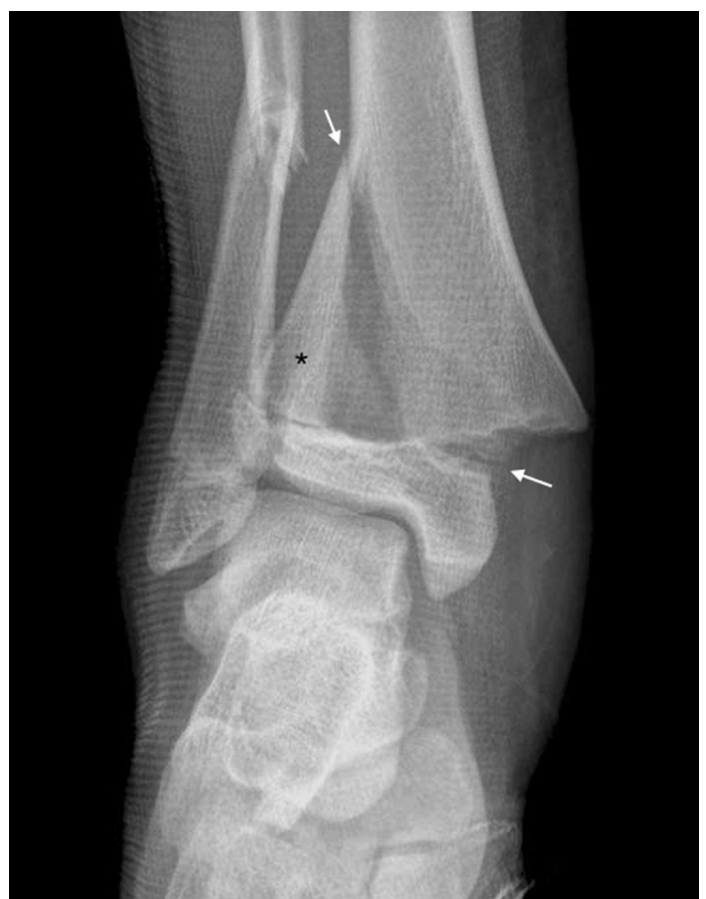

Fig. 4 A Salter-Harris Type II fracture of the distal tibia is shown. The path of the fracture is from the lateral metaphysis to the medial physis (white arrows). The resulting metaphyseal Thurston-Holland fragment is denoted by the asterisk.

fracture is more common in younger patients with a thicker physis.

Salter-Harris Type II (Figs. 2, 4) fractures are the most common type, accounting for $74 \%$ of physeal fractures. The fracture line enters in the plane of the physis and exits through the metaphysis. The separate metaphyseal fragment created is known as a Thurston-Holland fragment.

Salter-Harris Type III (Figs. 2, 5) fractures also enter in the plane of the physis, but exit through the epiphysis, resulting in an intraarticular fracture. Type III fractures are much less common than Type II fractures, however, potential sequelae include posttraumatic arthritis in addition to growth arrest.

Salter-Harris Type IV (Figs. 2, 6) fractures cross the physis, extending through the epiphysis and metaphysis. This fracture pattern disrupts the physis and articular surface and has an element of longitudinal instability. In addition to the risk of complete physeal arrest, a longitudinally malreduced Type IV fracture may result in the formation of a transphyseal bony bar with subsequent asymmetric growth or growth deformity.

Finally, Salter-Harris Type V (Figs. 2, 7) fractures are crush injuries at the physis as a result of compressive forces. These fractures are rare, and some authors, such as Peterson and Burkhart [18], have questioned their existence, but others, including Rathjen and Birch [22], accept

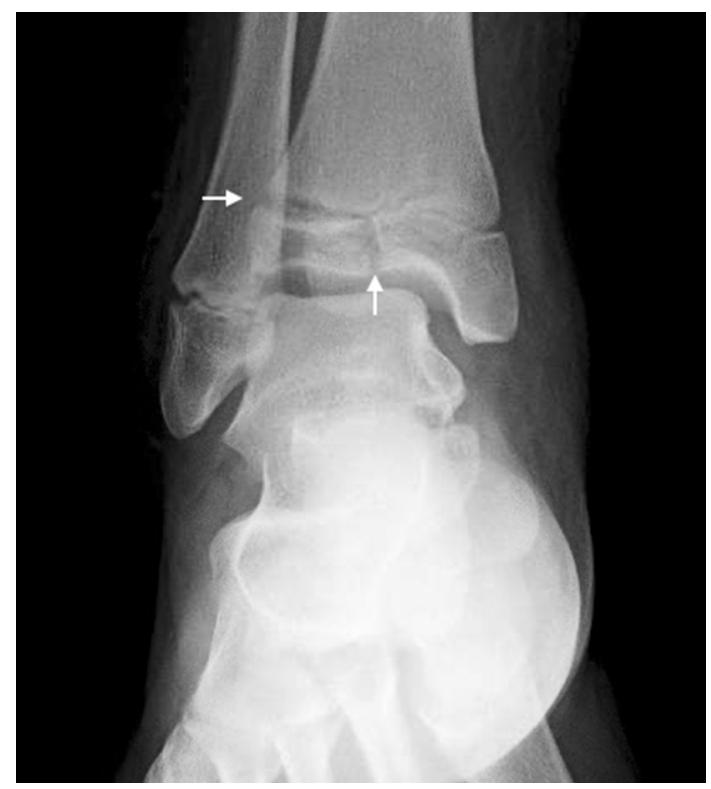

Fig. 5 This radiograph shows a Salter-Harris Type III facture of the distal tibia. The fracture is intraarticular, exiting through the epiphysis (white arrows).

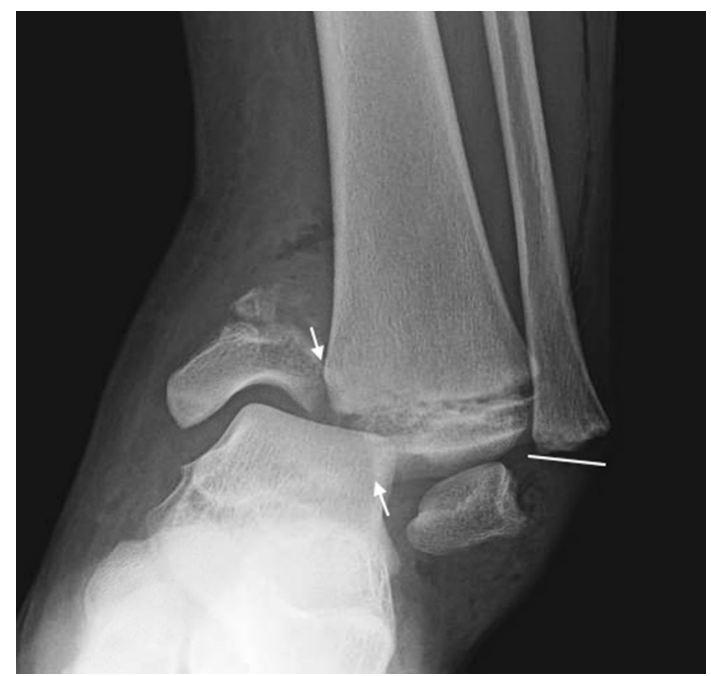

Fig. 6 The injury at the distal tibial sustained by a pediatric patient is a Salter-Harris Type IV fracture (white arrows) and can be seen traveling from the medial metaphysis to exit through the epiphysis. The injury at the distal fibula is a Salter-Harris Type I fracture (white line).

them as a distinct etiology. This type of compressive damage to the physis also can occur as a stress injury and can be seen in gymnasts with repetitive loading on an extended wrist [4].

To understand the pathomechanics of a physeal fracture, a basic understanding of growth plate histology is necessary. Salter and Harris [26] performed extensive histologic analysis of normal physeal anatomy, fracture patterns at the physis, and physeal healing after fracture. 


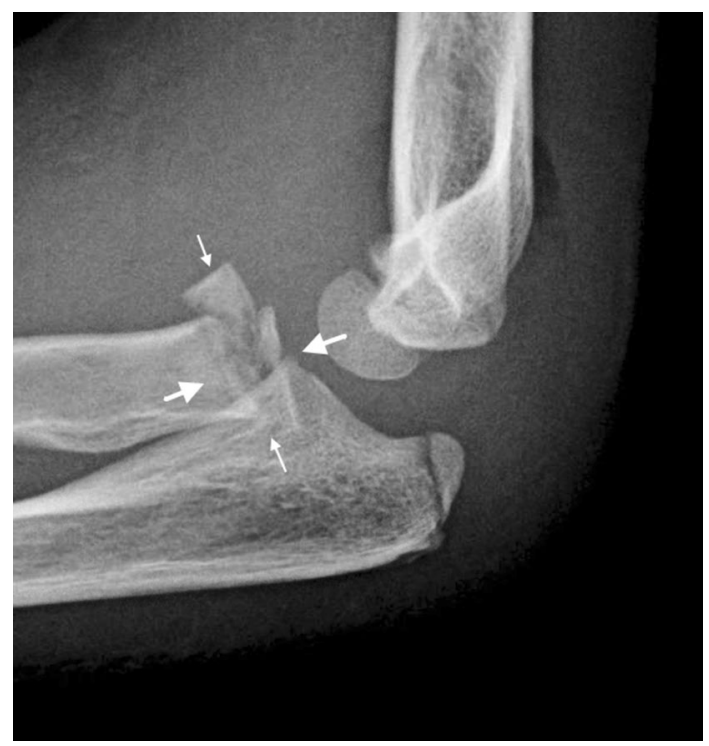

Fig. 7 This radiograph shows a Salter-Harris Type V fracture or crush injury to the physis of the proximal radius. The smaller arrows show the path of the fracture while the larger arrows represent the compressive force causing the injury.

The physis can be subdivided into four different zones, starting from the epiphysis and extending to the metaphysis (Fig. 1). Zone 1 is the "resting zone" and is located adjacent to the epiphysis and contains resting cells or germinal matrix, largely composed of relatively metabolically inactive chondroblasts. Zone 2 is the "proliferative zone" and contains more active chondrocytes that produce extracellular matrix proteins. Zone 3 is the "hypertrophic zone" and contains chondrocytes that are larger and more organized, but have decreased production of extracellular matrix proteins. This zone often is broken into three subzones: the zone of maturation, the zone of degeneration, and the zone of provisional calcification. The zone of provisional calcification constitutes a transitional area between calcified and noncalcified extracellular matrix proteins, effectively making this zone the weakest [24]. Through histologic analysis, Salter and Harris showed that fracture propagation and physeal separation typically occur at this level. Zone 4 is the final layer-the "zone of calcification"-where cartilage is calcified and begins to be remodeled into bone.

The physis is encircled at is periphery by fibrocartilaginous tissue that includes the groove of Ranvier and the ring of LaCroix (Fig. 1). The groove of Ranvier is a microscopic stricture at the diaphyseal end of the physis. It contains chondroblasts, osteoblasts, and fibroblasts that support the peripheral growth of the physis. The ring of LaCroix is a strong fibrous structure that overlies the groove of Ranvier and connects the epiphyseal periosteum to the metaphyseal periosteum, adding stability to the physis [22].
Salter and Harris [26] reported that, in the majority of physes, the blood supply to the proliferating cells arises from the epiphysis via its periosteum. Since the zone of provisional calcification is metaphyseal relative to the proliferating cells of the physis, epiphyseal blood supply theoretically remains intact with Types I and II fractures. Conversely, Types III and IV fractures exit epiphyseal, violating and potentially devascularizing the proliferating cell layer. Salter and Harris recognized that certain physes were especially prone to devascularization, namely the femoral and radial head. The epiphyses in these locations are completely covered by articular cartilage and have no periosteal blood supply. Alternately, the blood supply is metaphyseal and laterally traverses the rim of the physis, easily disrupted by the shear forces seen in a Type 1 fracture [6].

This model provides a framework to think about the types of physeal fractures, however, clinical reality is somewhat more complex. Subsequent histologic studies have shown that, depending on the forces involved, physeal injuries commonly involve multiple layers of the physis and rarely are isolated to the zone of provisional calcification [9, 14]. This is clinically evident with Type II fractures. These fractures can result in growth arrest despite theoretically leaving the proliferating cells and their blood supply intact. Jaramillo et al. [9] reported that MRI has the ability to elucidate which physeal zones are involved in an injury, allowing for better understanding of the growth plate injury.

\section{Validation}

Although the Salter-Harris classification is in common use, there are relatively few formal validation studies. Thawrani et al. [30] examined the intra- and interobserver reliability of classifying pediatric distal tibia fractures and found very high rates of intraobserver reliability and fairly robust rates of interobserver reliability (Kappa coefficient, 0.57-0.67). Several studies have compared the ability to classify fractures involving the growth plate on plain radiographs versus three-dimensional (3-D) imaging [11, 19, 27]. These studies do not specifically use interobserver reliability as an endpoint, instead showing that fracture displacement is consistently underappreciated on plain radiographs [11, 19], and that 3-D imaging can better elucidate fracture patterns and change the classification of the fracture [27].

A high rate of interobserver reliability of the classification is important to its clinical utility. Despite a lack of formal validation, the Salter-Harris classification has stood the test of time and is in widespread use. One may speculate that this prevalence is attributable to its inherent simplicity and being nearly universally known in the 
orthopaedic community. There are limitations to using a poorly validated classification system and there may be some benefit to perform additional validation studies of the Salter-Harris classification.

\section{Limitations}

The most significant limitation, as discussed above, is a paucity of studies formally validating the Salter-Harris classification, including interobserver reliability, intraobserver reliability, and accuracy in predicting fracture behavior. This validation is necessary to establish confidence in the classification and its implications. Lack of validation does not mean the classification is invalid, however, users should be aware of this limitation and use the classification accordingly. Future efforts to improve validation of the Salter-Harris classification could potentially resolve these concerns.

Another major limitation is that the Salter-Harris classification is not an independent predictor of a fracture's prognosis. It is tempting to equate physeal arrest with prognosis when discussing fractures involving the physis, however, physeal arrest is only one component and is of variable clinical significance depending on remaining growth and the location of the deformity. Salter and Harris [26] recognized the complexity of this issue and commented that prognosis was not related to fracture classification alone, but also to the age of the patient, preservation of blood supply, presence of an open fracture, method of reduction, intraarticular displacement, quality of reduction, method and length of immobilization, and, of particular importance, the specific physis involved. Even if the outcome is limited to the presence of growth arrest alone, many authors agree that the SalterHarris classification is not a good predictor of prognosis [2, 3, $7,12,23,29]$. For example, fractures involving the distal femoral physis tend to be high energy and have a rate of physeal arrest near $40 \%$ [2, 7]. Initial fracture displacement and accuracy of reduction have been found to be the most important prognostic indicators [2, 12]. Multiple studies examining physeal fractures at the distal tibia also have found that fracture displacement and mechanism of injury are the most significant prognostic indicators [10, 25, 28]. In a study of distal radius fractures, Cannata et al. [5] found that the rate of physeal arrest at the distal radius was less than $30 \%$ while the rate of physeal arrest at the distal ulna approached $80 \%$, however, neither was significantly correlated to Salter-Harris classification and fewer than $5 \%$ of patients had residual symptoms or functional deficits. The most commonly reported predictors of physeal arrest appear to be initial fracture displacement, mechanism of injury, and accuracy of reduction.

Discussion of the prognostic utility of the Salter-Harris classification highlights another significant limitation, which

\section{Peterson Classification}
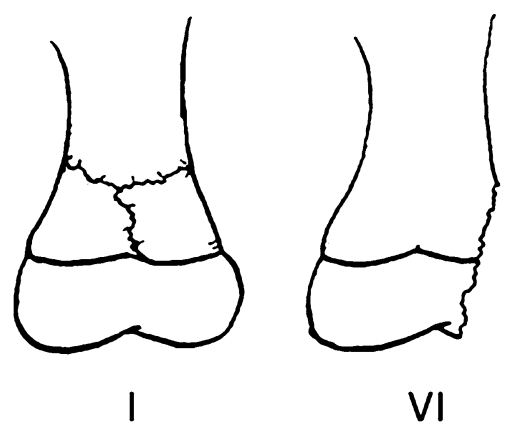

Fig. 8 The Peterson classification adds two new fracture variants to the Salter-Harris classification. A Peterson Type I fracture is transmetaphyseal with extension into the physis, and a Peterson Type VI fracture has loss of part of the physis and typically is described as an open "lawnmower" type of injury.

is lack of anatomic specificity. In their original article, Salter and Harris [26] recognized important variations in gross anatomy between different physes, however their proposed classification and discussion focused on the microanatomy of the generic physis. This prevents the classification from becoming overly complex; however, it limits the ability of the classification system to guide treatment or indicate prognosis with any specific fracture. This lack of specificity and comprehensiveness has been the impetus behind several subsequently proposed classification systems. In 1982, Ogden [15] proposed a classification scheme that expanded the Salter-Harris classification by adding four additional fracture types and multiple subtypes of each of the five original fracture types. The classification was meant to be applicable throughout the body, but rarely is used today, likely because of its complexity. In 1994, Peterson [17] proposed an expanded system based on an impressive epidemiologic study of physeal injuries. Peterson [16] added two new variants of physeal injuries (Peterson Types I and VI) (Fig. 8). A Peterson I injury is a metaphyseal fracture with extension into the physis (Fig. 9). A Peterson Type VI injury represents the loss of part of the physis. Peterson and Burkhart [18] also removed the Salter-Harris Type V fracture variant, questioning its existence; however, the omission of the Salter-Harris Type V fracture generally is not accepted. Rathjen and Birch [22] suggested maintaining the SalterHarris classification and adding Peterson Types I and VI as additional and unique entities.

\section{Conclusions and Uses}

The Salter-Harris classification continues to be relevant and serve an important purpose in orthopaedics despite 


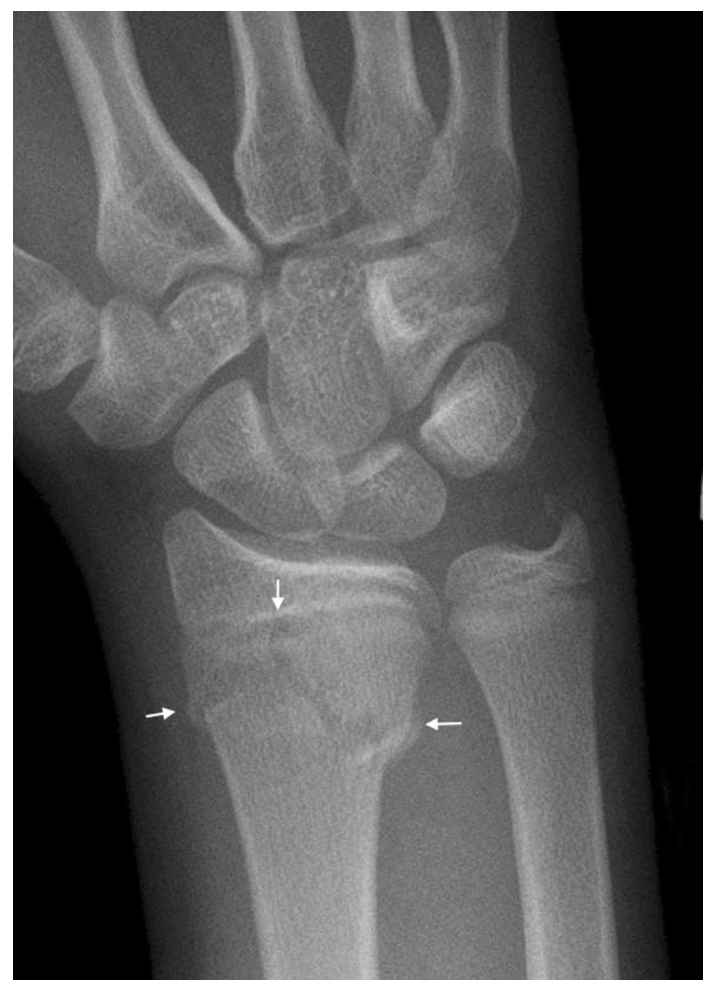

Fig. 9 This radiograph shows a Peterson Type I fracture of the distal radius. The fracture line is marked by arrows and can be seen crossing the metaphysis, and also extending into the physis.

substantial limitations. It is not a comprehensive system for classifying physeal injuries, guiding treatment, or determining prognosis. These limitation may be inherent to a classification that is intended to be generically applied to physeal fractures and does not attempt to account for anatomic variation between physes or unique clinical considerations of fractures in different locations. The Salter-Harris classification does provide a foundation to help clinicians understand how pediatric fractures relate to the anatomy and architecture of an open physis. Additionally, the generic nature of the classification allows it to be extremely simple and widely applied. The Salter-Harris classification has become part of the language used in orthopaedics, is nearly universally understood, and is used by orthopaedic practitioners, greatly facilitating communication. This is where the classification derives much of its utility. It may be more appropriate to think of the SalterHarris classification as descriptive terminology with general clinical implications than a specific fracture classification that is expected to dictate treatment and prognosis. A thorough understanding of the scope of pediatric trauma and anatomy is necessary to guide treatment decisions and understand expected outcomes.

\section{References}

1. Aitken AP. The end results of the fractured distal tibial epiphysis. J Bone Joint Surg Am. 1936;18:685-691.

2. Arkader A, Warner WC Jr, Horn BD, Shaw RN, Wells L. Predicting the outcome of physeal fractures of the distal femur. $J$ Pediatr Orthop. 2007;27:703-708.

3. Bassett FH 3rd, Goldner JL. Fractures involving the distal femoral epiphyseal growth line. South Med J. 1962;55:545-547.

4. Caine D, Roy S, Singer KM, Broekhoff J. Stress changes of the distal radial growth plate: a radiographic survey and review of the literature. Am J Sports Med. 1992;20:290-298.

5. Cannata G, De Maio F, Mancini F, Ippolito E. Physeal fractures of the distal radius and ulna: long-term prognosis. J Orthop Trauma. 2003;17:172-179; discussion 179-180.

6. Dale GG, Harris WR. Prognosis of epiphyseal separation: an experimental study. J Bone Joint Surg Br. 1958;40:116-122.

7. Eid AM, Hafez MA. Traumatic injuries of the distal femoral physis: retrospective study on 151 cases. Injury. 2002;33:251255.

8. Foucher JT. De la divulsion des epiphyses. Cong Med France. 1863;1:63-72.

9. Jaramillo D, Kammen BF, Shapiro F. Cartilaginous path of physeal fracture-separations: evaluation with MR imaging-an experimental study with histologic correlation in rabbits. Radiology. 2000;215:504-511.

10. Leary JT, Handling M, Talerico M, Yong L, Bowe JA. Physeal fractures of the distal tibia: predictive factors of premature physeal closure and growth arrest. J Pediatr Orthop. 2009;29:356361.

11. Lippert WC, Owens RF, Wall EJ. Salter-Harris type III fractures of the distal femur: plain radiographs can be deceptive. $J$ Pediatr Orthop. 2010;30:598-605.

12. Lombardo SJ, Harvey JP Jr. Fractures of the distal femoral epiphyses: factors influencing prognosis: a review of thirty-four cases. J Bone Joint Surg Am. 1977;59:742-751.

13. Mizuta T, Benson WM, Foster BK, Paterson DC, Morris LL. Statistical analysis of the incidence of physeal injuries. $J$ Pediatr Orthop. 1987;7:518-523.

14. Moen CT, Pelker RR. Biomechanical and histological correlations in growth plate failure. J Pediatr Orthop. 1984;4:180-184.

15. Ogden JA. Injury to the growth mechanisms of the immature skeleton. Skeletal Radiol. 1981;6:237-253.

16. Peterson HA. Physeal fractures: Part 2. Two previously unclassified types. J Pediatr Orthop. 1994;14:431-438.

17. Peterson HA. Physeal fractures: Part 3.Classification. J Pediatr Orthop. 1994;14:439-448.

18. Peterson HA, Burkhart SS. Compression injury of the epiphyseal growth plate: fact or fiction? J Pediatr Orthop. 1981;1:377-384.

19. Petit P, Panuel M, Faure F, Jouve JL, Bourliere-Najean B, Bollini $G$, Devred P. Acute fracture of the distal tibial physis: role of gradient-echo MR imaging versus plain film examination. AJR Am J Roentgenol. 1996;166:1203-1206.

20. Poland J. Traumatic Separation of the Epiphyses. London, England: Smith, Elder \& Co; 1898.

21. Rang M. The Growth Plate and Its Disorders. Harcourt Brace/ Churchill Livingstone; 1968.

22. Rathjen KE, Birch JG. Physeal injuries and growth disturbances. In: Beaty JH, Kasser JR, eds. Rockwood and Wilkins' Fractures in Children. 7th ed. Philadelphia, PA: Lippincott Williams \& Wilkins; 2010:91-119.

23. Riseborough EJ, Barrett IR, Shapiro F. Growth disturbances following distal femoral physeal fracture-separations. $J$ Bone Joint Surg Am. 1983;65:885-893. 
24. Rogers LF. The radiography of epiphyseal injuries. Radiology. 1970;96:289-299.

25. Rohmiller MT, Gaynor TP, Pawelek J, Mubarak SJ. Salter-Harris I and II fractures of the distal tibia: does mechanism of injury relate to premature physeal closure? $J$ Pediatr Orthop. 2006;26:322-328.

26. Salter RB, Harris WR. Injuries involving the epiphyseal plate. $J$ Bone Joint Surg Am. 1963;45:587-622.

27. Smith BG, Rand F, Jaramillo D, Shapiro F. Early MR imaging of lower-extremity physeal fracture-separations: a preliminary report. J Pediatr Orthop. 1994;14:526-533.
28. Spiegel PG, Cooperman DR, Laros GS. Epiphyseal fractures of the distal ends of the tibia and fibula: a retrospective study of two hundred and thirty-seven cases in children. J Bone Joint Surg Am. 1978;60:1046-1050.

29. Stephens DC, Louis E, Louis DS. Traumatic separation of the distal femoral epiphyseal cartilage plate. J Bone Joint Surg Am. 1974;56:1383-1390.

30. Thawrani D, Kuester V, Gabos PG, Kruse RW, Littleton AG, Rogers KJ, Holmes L, Thacker MM. Reliability and necessity of computerized tomography in distal tibial physeal injuries. $J$ Pediatr Orthop. 2011;31:745-750. 